\title{
¿Cómo perciben los estudiantes el clima de aprendizaje en el aula invertida en cirugía?: Lecciones aprendidas y recomendaciones para su implementación
}

\author{
Luis Domínguez T. ${ }^{1}$, Álvaro Sanabria Q. ${ }^{1}$ y Diego Sierra B. ${ }^{1}$
}

'Departamento de Cirugía, Universidad de la Sabana, Chía, Colombia.

Recibido el 27 de julio de 2017 y aceptado para publicación el 12 de septiembre de 2017.

Correspondencia a: Dr. Luis Carlos Domínguez carlosdot@unisabana.edu.co
How do students perceive the learning climate during the inverted classroom in surgery? Lessons learned and recommendations for their implementation

Introduction/Aims: The use of the inverted classroom as a teaching approach in the surgical curriculum is under development. There is a need for studies informing about their effectiveness and relationship with the learning climate. The aim of this study is to present the accumulated measurement of the learning climate in the inverted classroom in surgery, and share some recommendations for its implementation. Material and Method: The Dundee Ready Educational Environment Measure (DREEM) evaluated the student's perceptions about the learning climate. The overall score of instrument, as its five domains were calculated. Results: A total of 444 students were included. The overall DREEM score indicated a learning climate of excellence. In general, teaching was well perceived by the students, who felt confident about their academic abilities, perceived their teachers as models, identified a positive attitude in the learning atmosphere, and perceived an acceptable social environment. Discussion: The approach of inverted classroom in surgery is associated with a productive learning climate. These results provide new evidence to the high levels of student satisfaction and adequate perceptions about student self-confidence for learning in surgery showed in other studies. Conclusions: The longitudinal measurement of the learning climate in surgery by using the inverted classroom model demonstrates scores of excellence in the evaluated population. Further studies are needed to explore in depth their effects on learning and cognitive abilities in students.

Key words: inverted classroom; surgery; students; learning climate; Colombia.

\section{Resumen}

Introducción/objetivos: El uso del aula invertida como enfoque de enseñanza en el currículo de cirugía se encuentra en una etapa inicial de desarrollo. Existe la necesidad de estudios que soporten su efectividad y demuestren su relación con el clima de aprendizaje. El objetivo de este estudio es presentar la medición longitudinal (2013-2016) del clima de aprendizaje en el aula invertida en cirugía, y ofrecer algunas recomendaciones para su implementación. Material y Método: Las percepciones del clima de aprendizaje fueron evaluadas mediante el instrumento Dundee Ready Educational Enviroment Measure (DREEM). Se calculó el puntaje global del instrumento, así como el de cada uno de sus cinco dominios. Resultados: Un total de 444 estudiantes fueron incluidos. El puntaje global del DREEM indicó un clima de aprendizaje de excelencia. En general la enseñanza fue bien evaluada por los estudiantes, quienes se sintieron seguros de sus habilidades académicas, percibieron a sus profesores como modelo, identificaron una actitud positiva en la atmosfera, y percibieron el ambiente social como aceptable. Discusión: El enfoque de enseñanza de aula invertida en cirugía se asocia con un clima de aprendizaje productivo. Estos resultados aportan nueva evidencia a los altos niveles de satisfacción estudiantil y adecuadas percepciones sobre la autoconfianza estudiantil para el aprendizaje en cirugía demostrados en otros estudios. Conclusiones: La medición longitudinal del clima de aprendizaje en cirugía, utilizando el modelo de aula invertida demuestra puntaciones de excelencia en la población evaluada. Se requieren nuevos estudios que exploren en profundidad sus efectos sobre el aprendizaje y las habilidades cognitivas en los estudiantes.

Palabras clave: aula invertida; cirugía; estudiantes; clima de aprendizaje; Colombia. 


\section{Introducción}

El Aula Invertida es un enfoque de enseñanza revolucionario frente al enfoque tradicional de clases magistrales. Es un enfoque constructivista, que invierte los componentes tradicionales de una clase, y los distribuye en actividades "fuera del aula" en forma de videos, documentos y ejercicios de autoaprendizaje, entre otros, y que posteriormente utiliza el tiempo "en el aula" para ejercicios de aprendizaje activo $^{1}$. Como objetivo de aprendizaje, este enfoque persigue que el estudiante desarrolle habilidades cognitivas superiores de análisis, aplicación y evaluación del conocimiento, en vez de simplemente memorizar datos y hechos puntuales. La educación de pregrado en medicina no es ajena a esta transformación, y en los últimos años ha aumentado su uso en diversas asignaturas a lo largo del currículo. No obstante, la experiencia en cirugía es aún incipiente con respecto a otras áreas del pregrado, y hasta ahora se conocen algunos resultados sobre su efectividad, por una parte con respecto a desenlaces académicos y de satisfacción estudiantil, y por otra con relación al clima de aprendizaje.

En cuanto a los primeros, en un reciente estudio transversal de métodos mixtos realizado en la Universidad de Stanford (California), se exploraron las percepciones de los estudiantes de medicina sobre el modelo de aula invertida durante su rotación por cirugía. Este estudio demostró altos niveles de satisfacción estudiantil y adecuadas percepciones sobre la confianza de los estudiantes para su rotación clínica y la preparación de exámenes nacionales de la NBME (National Board of Medical Examiners) ${ }^{2}$. En los resultados cualitativos, los autores identificaron que el enfoque de aula invertida, permitió la creación de un ambiente de aprendizaje único, interactivo, atractivo e innovador, el cual proporcionó "tiempo protegido" para el autoaprendizaje. Asimismo, en otra publicación, realizada por los mismos autores, se comparó una cohorte histórica de estudiantes que habían recibido enseñanza tradicional (clase magistral), y otros que iniciaron un enfoque de aula invertida en cirugía, y se calcularon los puntajes, pre y post-test, con respecto a la satisfacción estudiantil y al interés en la cirugía como especialidad (ambos más altos con el enfoque de Aula invertida, $\mathrm{p}<0,001)$. Sin embargo, no se hallaron diferencias significativas sobre el efecto de cada uno de los enfoques en los puntajes de los exámenes nacionales de la $\mathrm{NBME}^{3}$. Por otra parte, con respecto al clima de aprendizaje, un reciente estudio comparó las percepciones de 207 estudiantes acerca del clima del aula invertida en cirugía, y el que percibían de las clases magistrales durante su carrera, mediante el instrumento Dundee Ready Education Environment Measure (DREEM). Los autores identificaron mejores puntajes globales, y de todos los dominios del instrumento, con el enfoque de aula invertida en relación con un modelo enfocado en clases magistrales $(\mathrm{p}<0,001)^{4}$. Desafortunadamente este estudio no comparó directamente el enfoque tradicional de clases magistrales frente al del aula invertida en la misma asignatura.

Teniendo en cuenta este limitado número de estudios y sus debilidades, existe la necesidad de realizar investigaciones con un mayor número de participantes que permitan comparar el enfoque de enseñanza de aula invertida en cirugía contra otros enfoques tradicionales y no tradicionales, correlacionarlo con mediciones objetivas de aprendizaje y habilidades cognitivas, y realizar estudios de seguimiento del clima de aprendizaje luego de su estandarización ${ }^{2-4}$. El presente estudio responde a este último llamado. Nuestro objetivo es presentar la medición acumulada del clima de aprendizaje en el enfoque de aula invertida tras cuatro años de estandarización en el marco de un currículo tradicional. Fruto del aprendizaje, en este estudio se presentan algunas recomendaciones prácticas para su implementación, las cuales podrían contribuir a la transformación de los arraigados enfoques tradicionales de enseñanza vigentes en la educación quirúrgica, que obligan a pensar en estrategias educativas diferentes en el siglo $\mathrm{XXI}^{5}$.

\section{Materiales y Métodos}

\section{Intervención}

A partir del año 2013 se inició un proceso de revisión del enfoque de enseñanza en el curso de cirugía para estudiantes de pregrado de la Facultad de Medicina de la Universidad de la Sabana (Colombia). Los cambios buscaban ofrecer una respuesta a la necesidad percibida de proveer herramientas para el aprendizaje autorregulado, fortalecer el pensamiento crítico y la toma de decisiones en los estudiantes. De acuerdo al perfil demográfico y epidemiológico colombiano se llevaron a cabo ajustes a los Resultados Esperados de Aprendizaje (REA) de acuerdo al uso de dos metodologías validadas para el diseño curricular ${ }^{6,7}$, los cuales fueron avaladas en sus aspectos metodológicos y éticos por la Comisión de Educación Médica (Facultad de Medicina, Universidad de la Sabana). Posteriormente, el curso se distribuyó en 18 semanas, en cada una de las cuales se revisa un contenido temático por órganos 
y sistemas (por ejemplo, patología quirúrgica de la vesícula biliar y sistema biliar extrahepático). El enfoque de enseñanza de aula invertida incluyó el diseño de actividades "fuera" y "en el aula". Dentro de las primeras se desarrollaron 65 clases teóricas en video (de duración aproximada de 10-15 min), documentos, lecturas recomendadas, casos clínicos para desarrollo individual y un foro para discusión. Estos recursos educativos se cargaron en una plataforma virtual institucional, la cual se revisa periódicamente. El tiempo de autoestudio es de 10$15 \mathrm{~h} /$ semana aproximadamente. Posteriormente se diseñaron las actividades "en el aula", las cuales se desarrollan durante 3 h/semana. En cada actividad, se utiliza un enfoque interactivo de aprendizaje en pequeños grupos de estudiantes para el análisis de casos clínicos. Los profesores realizan tutoría de estos grupos y luego actúan como facilitadores de

Tabla 1. Guía para la interpretación de los resultados globales del DREEM y sus dominios

\section{Puntuación global}

0-50 puntos: Ambiente educativo muy pobre

51-100 puntos: Ambiente educativo con muchos problemas

101-150 puntos: Ambiente educativo con más aspectos positivos que negativos 151-200 puntos: Ambiente educativo excelente

\section{Puntuación por dominios}

Dominio 1 (percepción de los estudiantes sobre el aprendizaje) 0-12: muy pobre

13-24: la enseñanza se percibe negativamente

25-36: percepción más bien positiva de la enseñanza

37-48: la enseñanza es altamente estimulante

Dominio 2 (percepción de los estudiantes sobre el papel de los profesores) 0-11: abismal (pésimo)

12-22: necesitan entrenamiento educacional

23-33: encaminados en la dirección correcta

34-44: docentes modelos

Dominio 3 (percepción de los estudiantes sobre sus habilidades académicas)

0 - 8: sentimiento de fracaso total

9-16: muchos aspectos negativos

17-24: sintiéndose más en el lado positivo

25-32: seguros

Dominio 4 (percepción de la atmósfera de aprendizaje)

0-11: ambiente pésimo

12-24: hay muchos aspectos que necesitan cambiar

25-36: hay una atmósfera positiva

37-48: percepción general buena

Dominio 5 (percepción del entorno social)

0 - 7: miserable

8-14: no es un buen lugar

15-21: no demasiado mal

22-28: muy bien socialmente

Fuente: Med Teach, 2005;27:326-331; Educ Health (Abingdon). 2009;22:11. la discusión sobre los mismos en una plenaria. En total se analizan cerca de 80 casos clínicos/semestre. La evaluación sumativa se realiza mediante dos exámenes de progreso y uno final.

\section{Mediciones, participantes y análisis estadístico}

La medición del clima de aprendizaje, definido como las percepciones que tienen los estudiantes sobre las características formales e informales de un ambiente educativo ${ }^{8,9}$, fueron evaluadas mediante la versión en español del instrumento Dundee Ready Educational Enviroment Measure (DREEM) ${ }^{8}$. La medición se realizó de forma anónima, voluntaria y confidencial por los participantes (estudiantes de cirugía), nueve semanas luego de iniciar cada curso. Esta medición se realizó con el fin de monitorizar rutinariamente la calidad del clima de aprendizaje en cada cohorte de estudiantes que cursó la asignatura de cirugía desde el primer semestre de 2013 hasta el segundo semestre de 2016. El instrumento está compuesto por cinco dominios que componen el clima de aprendizaje: 1) percepción de los estudiantes sobre su aprendizaje (12 ítems); 2) percepción de los estudiantes sobre sus habilidades académicas (8 ítems); 3 ) percepción que los estudiantes sobre el papel de los profesores (11 ítems); 4) percepción de la atmosfera de aprendizaje (12 ítems); 5) percepción del ambiente social (7 ítems). Las propiedades psicométricas de la versión en español del DREEM y sus dominios son adecuadas (coeficiente alfa de Cronbach $>, 90)^{4,10}$. El DREEM consta de 50 preguntas que son calificadas mediante una escala de Likert (0: totalmente en desacuerdo; 4: totalmente de acuerdo). El puntaje máximo del DREEM es de 200 puntos, resultante de la multiplicación de todas las preguntas por la calificación máxima de la escala de Likert. Algunas de las preguntas tienen una calificación inversa de acuerdo al enunciado (preguntas $11,12,19,20,21,23,42,43,46)$. La escala global, así como cada uno de sus dominios da origen a un puntaje específico el cual se puede agrupar por rangos que indican pobres o muy buenas percepciones, respectivamente (Tabla 1). La información se recolectó en un cuestionario impreso y se almacenó en una base de datos en Excel (Microsoft Corp). Los datos se analizaron mediante el software Stata 13 (Stata Corp, US) y se presentaron como medias \pm desviaciones estándar (DE) y rangos.

\section{Resultados}

Durante el período de observación, se han llevado a cabo un total de ocho cursos semestrales de cirugía involucrando 480 estudiantes $(37,6 \%$ del género 


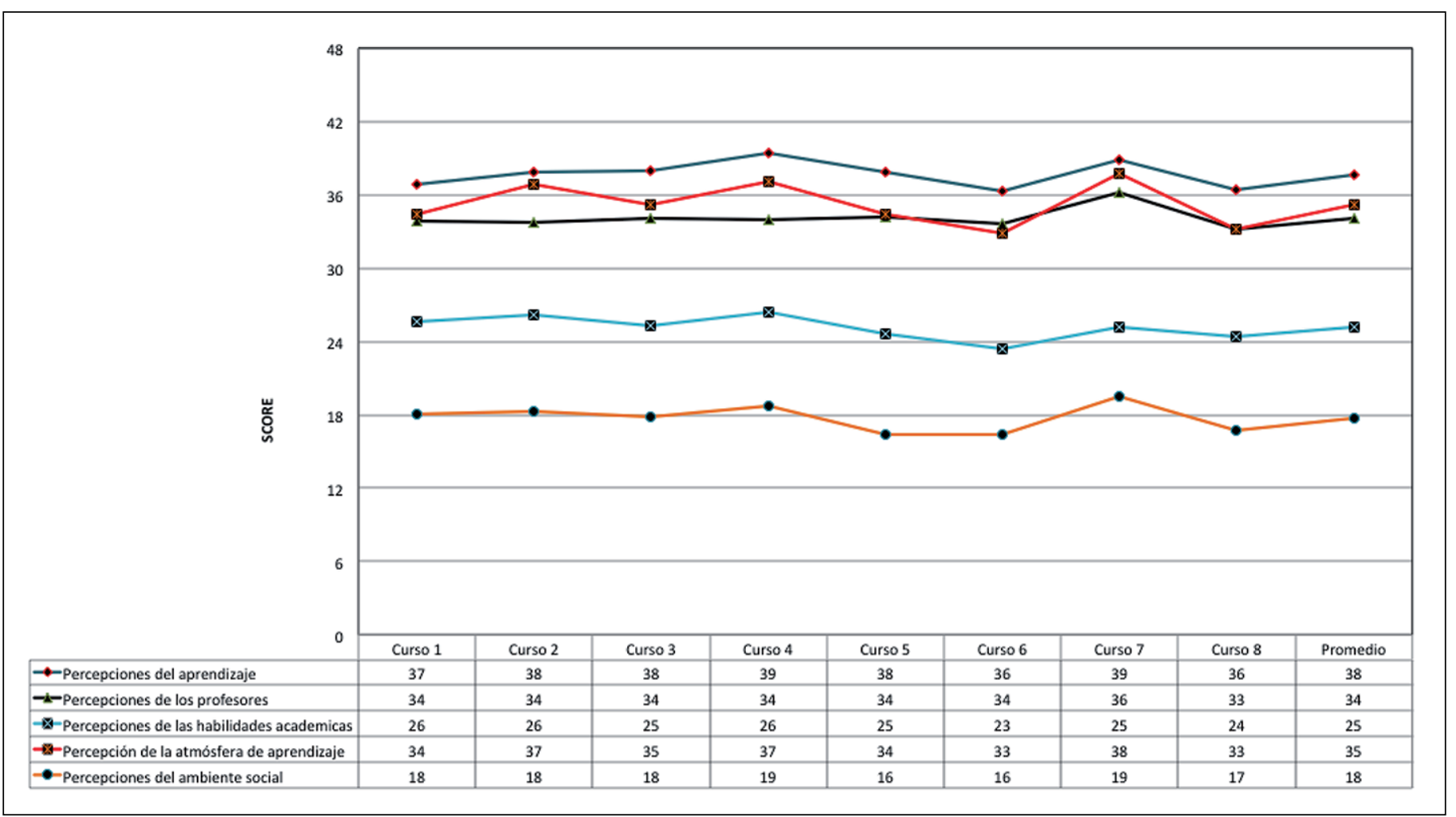

Figura 1. Esta figura muestra los puntajes por cada dominio del Dundee Ready Educational Environment en los ocho cursos de cirugía (2013-2016) empleando el modelo de aula invertida.

masculino). La tasa de respuesta final del instrumento DREEM fue de $92,5 \%$ (444 estudiantes). La edad promedio de los participantes fue $21,2 \pm 1,49$ años. La tasa de éxito académico fue de $97 \%$.

El coeficiente de confiabilidad del instrumento fue ,92. El puntaje global del DREEM fue 149,84 \pm 5,26 (143-157). El puntaje por dominios del DREEM fue: 1) percepción de los estudiantes sobre su aprendizaje: 37,69 $\pm 1,12$ (33-39); 2) percepción de los estudiantes sobre sus habilidades académicas (8 ítems); 25,13 \pm .99 (23-26); 3) percepción que los estudiantes sobre el papel de los profesores

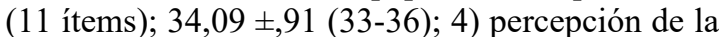
atmosfera de aprendizaje (12 ítems); 35,20 \pm 1.83 (33-38); 5) percepción del ambiente social (7 ítems): $17,73 \pm 1,13$ (16-19). La distribución del puntaje por dominios acumulado durante los ocho cursos se presenta en la Figura 1.

\section{Discusión}

Estrategias novedosas en los métodos de enseñanza en cirugía, como el enfoque de aula invertida, han sido poco exploradas en la educación en cirugía. La introducción de este tipo de estrategias requiere de evaluación continua, con el fin de determinar su utilidad y aplicabilidad, además de servir como modelo para modificaciones en otras asignaturas del currículo, y a su vez, en otras instituciones de educación superior. El objetivo de este estudio fue presentar los resultados de la medición acumulada del clima de aprendizaje de un enfoque de enseñanza de aula invertida, tras cuatro años de su implementación, en el curso de cirugía de pregrado en una universidad privada en Colombia. Los hallazgos indican que con este enfoque se logró mantener, a través del período de observación, un clima de aprendizaje cercano a la excelencia de acuerdo a los resultados globales del DREEM. No obstante, existen aún aspectos por mejorar, pues hay una distancia de aproximadamente 50 puntos con respecto a la máxima calificación. Así mismo los hallazgos indican que la enseñanza fue bien evaluada por los estudiantes (Dominio 1), los estudiantes se sintieron seguros de sus habilidades académicas (Dominio 2), los estudiantes percibieron a sus profesores como modelo (Dominio 3), se identificó una actitud posi- 
tiva en la atmósfera de aprendizaje (Dominio 4) y el ambiente social fue aceptable (Dominio 5).

Estos resultados pueden explicarse por la estandarización de un curso mediante metodologías validadas de diseño instruccional con resultados de aprendizajes esperados claramente definidos; por el mantenimiento de un entorno virtual "vivo" a través de las discusiones fluidas entre estudiantes y profesores; por el desarrollo profesoral focalizado en el uso de estrategias de enseñanza interactiva, mediante la discusión de casos clínicos, que permiten trasladar la teoría a la práctica; y por el seguimiento riguroso y el permanente monitoreo de su calidad a lo largo del tiempo. Consideramos que estos elementos explican los resultados de los primeros cuatro dominios del DREEM, y son comunes a los factores que favorecen un buen clima de aprendizaje en estudios previamente publicados ${ }^{11-17}$. Estos resultados tienen que ver con las ventajas que ofrece el aula invertida. En primer lugar, esta metodología aparta a los estudiantes y profesores de la monotonía y la rutina de la enseñanza magistral, enciclopédica y unidireccional y los conduce a un ambiente de discusión, en vez de uno de trasmisión de información. En segundo lugar, permite trasladar la teoría a la práctica. Finalmente, esta estrategia actúa como un punto de encuentro que ayuda a los estudiantes a contrastar la evidencia científica con la experiencia local que se vive en los hospitales e instituciones de salud en general, de acuerdo a los recursos disponibles, "las escuelas quirúrgicas" y los protocolos institucionales. Discutir interactivamente es una forma eficiente para mitigar el relativismo de conductas, $\mathrm{y}$ para que el estudiante se sitúe "en contexto", dentro de un núcleo central de conocimiento y la realidad local. Por otra parte, la calificación más baja entre los dominios estuvo asociada en la percepción de los estudiantes sobre el ambiente social. Este dominio explora aspectos como el soporte institucional para estudiantes con dificultades y la interacción social entre ellos. Es probable que este puntaje se explique por las exigencias propias de una rotación quirúrgica, la cual implica alta exigencia y compromiso asistencial que puede afectar las actividades sociales propias de los estudiantes. Otra explicación probable se debe a la distribución por grupos en los diferentes sitios de práctica, que puede romper lazos sociales previamente establecidos. Sin embargo, estas explicaciones hipotéticas distan del alcance de este estudio y requieren de nuevas investigaciones.

Este estudio ofrece una valiosa información prospectiva sobre el rendimiento de un enfoque de enseñanza cuidadosamente estandarizado y sus efectos en el clima de aprendizaje. Se resaltan en este estudio el diseño instruccional, la puesta en marcha y la medición de la calidad en una muestra representativa de estudiantes mediante el uso de un instrumento disponible validado universalmente. No obstante existen debilidades. Este estudio carece de una comparación directa con otras modalidades de enseñanza. Además, los resultados pueden ser afectados por la falacia ecológica, que no permite ver los avances individuales de los estudiantes.

El segundo objetivo de este estudio se relaciona con las implicaciones para la implementación efectiva de un enfoque de aula invertida (implicaciones prácticas), derivadas de nuestra experiencia, las cuales se pueden incluir en las siguientes diez recomendaciones:

1. Diseñar un aula invertida no es un proceso de la noche a la mañana. Toma tiempo y requiere apoyo institucional, y puede llegar a ser dispendioso si se hace bien. Requiere un adecuado manejo del cambio y liderazgo, pues puede amenazar el statu quo y la zona de confort en la que se encuentran tanto profesores como estudiantes. Es importante indagar por experiencias previas, institucionales o en el medio local, nacional o regional, con el fin de superar los desafíos que otros ya han resuelto, y aprender de su experiencia. El apoyo institucional es fundamental, pues requiere inversiones en entornos virtuales y espacios físicos para que funcione adecuadamente.

2. La aceptación del cambio por parte de los estudiantes es garantía de éxito. Parte de este cambio implica el fomentar en ellos, desde el principio y a lo largo del curso, la importancia de la autodeterminación en el aprendizaje. El cambio de modelo, desde uno tradicional hacia uno de tipo interactivo, implica ayudar a los estudiantes a derribar las barreras del paternalismo y la dependencia, y a fomentar la autonomía, competencia e interacción.

3. Es recomendable que el modelo de diseño instruccional incluya resultados esperados de aprendizaje que involucren taxonomías de conocimiento de niveles superiores (por ejemplo analizar, aplicar, evaluar), las cuales cuenten con información de soporte y procedimental claramente dispuesta en objetivos para las actividades "en el aula" y fuera del "aula". Igualmente, este diseño, permitirá que los resultados esperados de aprendizaje se ajusten con el sistema de evaluación. Estos elementos deben ser explícitos para los estudiantes.

4. Las actividades por "fuera del aula" no deben evitar un entorno virtual frío y lúgubre. El uso de estos entornos debe ser un punto de encuentro 
entre estudiantes y profesores a través de foros de discusión que mantengan "vivo" el ambiente y que den al estudiante la satisfacción de contar con supervisión apropiada a distancia. Igualmente, los materiales allí alojados deber ser claros, explícitos y suficientes. No es recomendable que este entorno se sobrecargue de materiales irrelevantes y tediosos. Recomendamos realizar videos concretos, bien preparados y de duración no mayor a $10 \mathrm{~min}$.

5. Las estrategias de trabajo interactivo "en el aula" son más importantes, en nuestro entendimiento y experiencia, que las actividades "fuera del aula", pues son precisamente las que fomentan el desarrollo de habilidades cognitivas avanzadas, diferentes a la capacidad de memorizar. No basta con desarrollar materiales virtuales, si estos no son incorporados en discusiones que permitan trasladar la teoría a la práctica. Recomendamos que las actividades interactivas cuenten con espacio físico y recursos adecuados. Por ejemplo, el uso de mesas circulares en donde discutan 8-10 estudiantes es fuertemente recomendable porque permite que ellos interactúen y discutan organizada y fácilmente.

6. Para el desarrollo exitoso de sesiones "en el aula", es importante incorporar estrategias de desarrollo profesoral centradas en el manejo de grupos pequeños, en reducir la carga cognitiva extraña y en aumentar la carga relevante e intrínseca. Los profesores son facilitadores de un proceso de construcción del aprendizaje por parte de los estudiantes. Por ejemplo, debe disminuirse al máximo que los profesores "tomen excesivamente la palabra", resuelvan los casos por los estudiantes, se tornen paternalistas e introduzcan conceptos irrelevantes o confusos, entre otros.

7. Es útil que las actividades "en el aula" sigan un orden establecido y que cada caso sea sintetizado apropiadamente con el fin que el estudiante identifique los puntos clave del análisis. Recomendamos incluir formatos como los mapas conceptuales o puntos clave al finalizar cada sesión, los cuales sean entregados a los estudiantes como nuevos materiales de estudio.

8. Es enriquecedor el abrir las aulas a invitados y profesores externos que fomenten y permitan el debate y la integración clínica. Recomendamos que durante las discusiones participen invitados de otras disciplinas y, en especial, en aquellos modelos de enseñanza tradicional, se inviten a profesores de las ciencias básicas y clínicas cuando sea necesario.
9. Los resultados de la aplicación de este enfoque de enseñanza deben medirse de forma periódica, grupal e individualmente. Una evaluación fundamental es el monitoreo de los aspectos relacionados con el clima de aprendizaje mediante el uso de instrumentos diseñados para tal fin.

10. Es importante que el uso de este, como de otros enfoques de enseñanza "no tradicional", esté alineado con el diseño de estudios que permitan fomentar la investigación en educación médica y la divulgación de sus resultados. Este aspecto contribuirá como insumo a la educación basada en la evidencia y permitirá transformar los esquemas de enseñanza.

Igualmente consideramos que cada una de estas diez recomendaciones abre la puerta para nuevas investigaciones en el futuro, las cuales se centran en aspectos relacionados con el manejo del cambio, el diseño instruccional, la enseñanza interactiva, la carga cognitiva, el desarrollo profesoral, la integración clínica y la evaluación permanente de la calidad, entre otros, todos ellos relacionados con los procesos de enseñanza y aprendizaje afines con el enfoque de aula invertida.

\section{Responsabilidades éticas}

Protección de personas y animales. Los autores declaran que los procedimientos seguidos se conformaron a las normas éticas del comité de experimentación humana responsable y de acuerdo con la Asociación Médica Mundial y la Declaración de Helsinki.

Confidencialidad de los datos. Los autores declaran que han seguido los protocolos de su centro de trabajo sobre la publicación de datos de pacientes.

Derecho a la privacidad y consentimiento informado. Los autores declaran que en este artículo no aparecen datos de pacientes.

\section{Financiación}

Facultad de Medicina, Universidad de la Sabana (Colombia).

\section{Conflicto de intereses}

Los autores declaran no tener ningún conflicto de intereses. 


\section{ARTÍCULO ORIGINAL}

\section{Bibliografía}

1. Moffett J. Twelve tips for "flipping" the classroom. Med Teach. 2015;37:331-6.

2. Liebert CA, Mazer L, Bereknyei Merrell S, Lin DT, Lau JN. Student perceptions of a simulation-based flipped classroom for the surgery clerkship: A mixed-methods study. Surgery. 2016;160:591-8.

3. Liebert CA, Lin DT, Mazer LM, Bereknyei S, Lau JN. Effectiveness of the Surgery Core Clerkship Flipped Classroom: a prospective cohort trial. Am J Surg. 2016;211:451-7

4. Domínguez LC, Vega NV, Espitia EL, Sanabria ÁE, Corso C, Serna AM, et al. Impact of the flipped classroom strategy in the learning environment in surgery: A comparison with the lectures. Biomedica 2015;35:513-21.

5. Tapia Jurado J. Challenges of surgery in the 21st century. Cir Cir. 2017;85:1-3.

6. Biggs J, Tang C. Teaching for Quality Learning at University. Society for Research into Higher Education \& Open University Press, McGraw-Hill 2007. UK

7. van Merriënboer JJG, Kirschner PA.
Ten Steps to Complex Learning: A Systematic Approach to Four-Component Instructional Design 2nd Edition Routledge, 2012.

8. Roff S, McAleer S, Skinner A. Development and validation of an instrument to measure the postgraduate clinical learning and teaching educational environment for hospital-based junior doctors in the UK. Med Teach 2005;27:326-31.

9. Delva MD, Kirby J, Schultz K, Godwin M. Assessing the relationship of learning approaches to workplace climate in clerkship and residency. Acad Med. 2004;79:1120-6.

10. Riquelme A, Oporto M, Oporto J, Méndez JI, Viviani P, Salech F, et al. Measuring students' perceptions of the educational climate of the new curriculum at the Pontificia Universidad Católica de Chile: performance of the Spanish translation of the Dundee Ready Education Environment Measure (DREEM). Educ Health (Abingdon) 2009;22:11.

11. DeRuisseau LR. The flipped classroom allows for more class time devoted to critical thinking. Adv Physiol Educ. 2016;40:522-8.

12. McLean S, Attardi SM, Faden L, Goldszmidt M. Flipped classrooms and student learning: not just surface gains. Adv Physiol Educ. 2016;40:47-55.

13. Ramnanan CJ, Pound LD. Advances in medical education and practice: student perceptions of the flipped classroom. Adv Med Educ Pract. 2017;13:63-73.

14. Morgan H, McLean K, Chapman C, Fitzgerald J, Yousuf A, Hammoud M. The flipped classroom for medical students. Clin Teach. 2015;12:155-60.

15. Rotellar C, Cain J. Research, Perspectives, and Recommendations on Implementing the Flipped Classroom. Am J Pharm Educ. 2016;80:34

16. Sharma N, Lau CS, Doherty I, Harbutt D. How we flipped the medical classroom. Med Teach. 2015;37:327-30.

17. McLaughlin JE, Roth MT, Glatt DM, Gharkholonarehe N, Davidson CA, Griffin LM, et al. The flipped classroom: a course redesign to foster learning and engagement in a health professions school. Acad Med. 2014;89:236-43. 